DOI: $10.1113 / E P 088106$

\title{
Commissioned Article (Symposium Report) \\ OXYGEN TRANSPORT AND UTILISATION DURING EXERCISE IN CYSTIC \\ FIBROSIS: CONTRIBUTORS TO EXERCISE INTOLERANCE
}

Zoe L. Saynor ${ }^{1 *}$, Mathieu Gruet ${ }^{2}$, Paula Rodriguez-Miguelez ${ }^{3}$, Ryan A. Harris ${ }^{4}$

Running Title: Exercising $\mathrm{O}_{2}$ transport and utilisation in cystic fibrosis

Keywords: cystic fibrosis; exercise intolerance; haemodynamics; $\mathrm{O}_{2}$ delivery; $\mathrm{O}_{2}$ utilisation

Word count: 3,056 (limit 3,000 words including in-text citations \& reference list)

References: 27 references

${ }^{1}$ School of Sport, Health and Exercise Science, Faculty of Science and Health, University of Portsmouth, UK.

${ }^{2}$ Toulon University, Laboratory of the Impact of Physical Activity on Health (IAPS), 83000 Toulon, France

${ }^{3}$ Department of Kinesiology and Health Sciences, College of Humanities and Sciences, Virginia Commonwealth University, USA.

${ }^{4}$ Georgia Prevention Institute, Department of Medicine, Augusta University, USA.

*Correspondence to Dr. Zoe L. Saynor, School of Sport, Health and Exercise Science, Faculty of Science and Health, University of Portsmouth, Portsmouth, Hampshire, UK, PO1 2ER. Tel: +44 (0)2392 843080 Email: zoe.saynor@port.ac.uk

This is an Accepted Article that has been peer-reviewed and approved for publication in Experimental Physiology, but has yet to undergo copy-editing and proof correction. Please cite this article as an Accepted Article; doi: 10.1113/EP088106.

This article is protected by copyright. All rights reserved. 


\section{NEW FINDINGS (symposium report)}

\section{- What is the topic of this review?}

This review highlights the central and peripheral mechanisms that alter oxygen transport and utilisation and thereby contribute to exercise limitation in people with cystic fibrosis, considering also viable therapeutic targets for intervention.

\section{- What advances does it highlight?}

Although traditionally considered a respiratory condition, pathological intramuscular and cardiovascular changes in people with cystic fibrosis appear to be key determinants of exercise intolerance up until the later stages of respiratory disease. Even young, habitually active patients with normal lung function experience multisystemic abnormalities, which play a role in exercise intolerance. 


\begin{abstract}
Cystic fibrosis (CF) is a complex condition, commonly associated with exercise limitation. The mechanisms responsible for this in CF are of interest, given that lower aerobic fitness is associated with an increased risk of being hospitalised with pulmonary exacerbation, a poorer quality of life and prognosis. Pathophysiological changes in lung function are considered central to CF, and may contribute to exercise limitation. However, it is now clear that the pathogenesis of exercise limitation in this population is multifactorial, with alterations in cardiovascular, muscle and pulmonary function contributing. Whilst some of these changes are attributable to respiratory disease per se, the CF transmembrane conductance regulator protein is also found in skeletal muscle and the vascular endothelium and can directly alter central and localised oxygen delivery, as well as the ability to effectively extract and utilise oxygen at the myocyte level. Since intense exercise poses considerable challenges to arterial oxygen content and/or blood flow and its supply to the working skeletal muscle, evaluating the exercise physiology of people with CF has helped us understand the mechanisms underlying exercise intolerance. Through several investigations over recent years, we have collectively demonstrated that people with $\mathrm{CF}$ exhibit reduced skeletal muscle oxygen extraction and utilisation during exercise, with a lesser contribution from haemodynamic or chronotropic mechanisms. Taken together, our findings highlight the importance of targeting mechanisms of skeletal muscle oxygen utilisation in CF to improve exercise tolerance and we offer potential therapeutic interventional strategies.
\end{abstract}

(238 / 300 words) 


\section{INTRODUCTION}

Cystic fibrosis (CF) is an autosomal recessive disease associated with defective or deficient expression of the CF transmembrane conductance regulator (CFTR) protein, a chloride and bicarbonate ion channel predominantly expressed in epithelial cells. CF pathophysiology is complex, affecting multiple organ systems including the lungs, pancreas, upper airway, liver, intestine, and reproductive organs. Traditionally, CF was characterised by lung congestion and infection and progressive respiratory failure. However, as clinical management and life expectancy improve, the importance of extrapulmonary manifestations is becoming increasingly apparent.

In this review, we highlight several disordered processes inherent to CF pathophysiology that can modulate oxygen $\left(\mathrm{O}_{2}\right)$ transport and utilisation. Finally, we consider the current and evolving potential therapeutic strategies that may ameliorate these alterations and convey both physiological and clinical benefits. Understanding and treating the mechanistic basis of impaired aerobic fitness is a clinical priority in people with $\mathrm{CF}$ (pwCF), since physical inactivity is associated with a poorer quality of life, increased hospitalisation risk, and higher rates of mortality.

\section{TO WHAT EXTENT DOES VENTILATORY LIMITATION CONTRIBUTE TO EXERCISE INTOLERANCE IN CYSTIC FIBROSIS?}

Exercise intolerance in pwCF was traditionally considered the result of poor pulmonary function and malnutrition. In the lungs, abnormally thick sticky mucus obstructs the airways and glands, leading to airway damage and alveoli collapse. These pathophysiological changes, typically characterising advanced lung disease, result in increased dead space ventilation and dynamic hyperinflation, exercise-induced ventilation-perfusion abnormalities, arterial desaturation and alveolar hypoventilation during exercise.

These abnormalities may compromise the ability of the lung to meet the ventilatory and gas exchange demands imposed by exercise and thus impair $\mathrm{O}_{2}$ delivery to the locomotor muscles. However, since the treatment of these pulmonary manifestations has considerably improved, neither ventilatory limitation or arterial hypoxemia are commonly observed in milder CF lung disease (even during maximal exercise). Indeed, recent investigations in physically active, well-nourished children and adolescents with preserved lung function have 
provided vital insight into the extrapulmonary factors that contribute to exercise intolerance in pwCF.

\section{OTHER PATHOPHYSIOLOGICAL ALTERATIONS THAT MAY IMPAIR $\mathrm{O}_{2}$ DELIVERY AND/OR $\mathrm{O}_{2}$ UTILISATION IN PEOPLE WITH CYSTIC FIBROSIS DURING EXERCISE}

Evidence is growing to support the existence of myocardial impairment in CF (Labombarda, 2016), even in youth (Giacchi et al., 2015; Eising et al., 2018), indicated by an increased pulmonary blood pressure (Giacchi et al., 2015) and diminished right and left ventricular function (Eising et al., 2018). CF-related chronic hypoxaemia, inflammation, infection and/or diabetes have been implicated, but CFTR dysfunction may also directly affect cardiac function since it is expressed in cardiac muscle (Hart et al., 1996; Chen et al., 2004). Moreover, CFTR can regulate cardiomyocyte contraction rate in neonatal mice and loss of CFTR in mice without lung pathology also leads to vascular remodelling and increased aortic stiffness (Sellers et al., 2013).

Increased arterial stiffness is also evident in adults and children with CF, even at a very young age (Eising et al., 2018). Recent data from our team also demonstrates peripheral micro- and macrovascular endothelial perturbations in young pwCF (Poore et al., 2013; Rodriguez-Miguelez et al., 2016). Questions remain regarding the mechanisms underlying vascular changes in CF, nevertheless CFTR has been linked, since it is expressed in human umbilical vein endothelial cells (Tousson et al., 1998), human pulmonary artery endothelial cells (Tousson et al., 1998), and human lung microvascular endothelial cells (HLMVECs). Furthermore, we recently demonstrated that CFTR is involved in the regulation of oxidative stress, inflammation and endothelial cell signalling in HLMVECs (Khalaf, 2020). Unpublished data from one of our laboratories, using the orbital shaker method, demonstrated that CFTR appears to regulate endothelial actin cytoskeleton dynamics and cellular alignment in response to flow, which is important for maintaining cellular integrity and promoting alignment with flow.

Skeletal muscle is also altered (Gruet et al., 2017). Reduced muscle mass and strength, particularly in the lower limbs, is common and not solely attributable to physical inactivity and malnutrition. $\mathrm{CF}$ is consistent with a scenario in which two major factors act synergistically to favour the development of skeletal muscle dysfunction: 1) more pulmonary/systemic inflammation leading to a greater exposure of the muscles to pro- 
inflammatory mediators, and 2) greater sensitivity of the pro-inflammatory mediator response to muscles (due to dysfunctional CFTR), which leads to exaggerated upregulation of atrophy pathways as well as impairment of contractile mechanisms (Gruet et al., 2017). Furthermore, reduced antioxidant capacity, due to increased systemic inflammation and/or oxidative stress, which we have recently phenotyped even in those with stable CF (Causer et al., 2020), may further impair mitochondrial efficiency and contribute to peripheral (and respiratory) muscle dysfunction. Similar to the cardiovascular system, CFTR is expressed in skeletal muscle (Lamhonwah et al., 2010) and clearly contributes to the pathophysiology; indicated by abnormal intracellular calcium and contractile responses to depolarisation in muscle with dysfunctional CFTR (Divangahi et al., 2009; Lamhonwah et al., 2010). CFTR has also been shown to mediate glutathione flux in epithelial cells (Linsdell \& Hanrahan, 1998) and thus plays a role in controlling the redox state, which is an important modifier of calcium release. Lack of CFTR has also been shown to predispose muscle atrophy and diaphragm muscle pump failure using a murine model (Divangahi et al., 2009).

\section{4. $\mathrm{O}_{2}$ DELIVERY AND UTILISATION DURING EXERCISE IN PEOPLE WITH CYSTIC FIBROSIS: RECENT ADVANCES IN OUR UNDERSTANDING}

The technological advancement of non-invasive monitoring systems, particularly those suitable for paediatric participants, recently permitted the study of the dynamic balance of $\mathrm{O}_{2}$ delivery-to-extraction in pwCF. Near-infrared spectroscopy (NIRS) and thoracic impedance cardiography have been used by our team and others to evaluate muscle oxygenation and oxidative capacity, an index of mitochondrial function, and cardiac function, respectively. In a series of experiments, we have concurrently measured the dynamics of pulmonary oxygen uptake $\left(\dot{\mathrm{V}}_{2}\right)$ and oxygenation within the microcirculation of exercising muscle, with additional measures of systemic $\mathrm{O}_{2}$ delivery, for the first time. This has allowed investigation into the dynamic balance between $\mathrm{O}_{2}$ delivery to and localised extraction and utilisation at the peripheral locomotor muscle of pwCF during exhaustive exercise, constant work rate exercise of different intensities, and in response to experimental manipulations of systemic $\mathrm{O}_{2}$ delivery.

Our investigation of the dynamic adjustments in localised muscle (vastus lateralis) fractional $\mathrm{O}_{2}$ extraction during exhaustive ramp incremental exercise in youth with mild-to-moderate CF lung disease (Saynor et al., 2014b), relative to pulmonary gas exchange and ventilation 
and systemic $\mathrm{O}_{2}$ delivery, was the first of its kind. The NIRS-derived muscle oxygenation signal provides insight into the local ratio of muscle $\mathrm{O}_{2}$ delivery-to-utilisation, with the deoxyhaemoglobin ([HHb]) signal considered a proxy for $\mathrm{O}_{2}$ extraction. This methodology permits inferences regarding blood flow within the microcirculation of exercising muscle. More rapid $[\mathrm{HHb}]$ dynamics typically occur in the face of reduced systemic or localised $\mathrm{O}_{2}$ delivery and vice versa. As expected, aerobic fitness was reduced in youth with mild-tomoderate $\mathrm{CF}$. However, despite impaired central $\mathrm{O}_{2}$ delivery (reduced $\mathrm{SpO}_{2}$ at exhaustion and $\mathrm{O}_{2}$ pulse, which were associated with $\left.\dot{\mathrm{V}} \mathrm{O}_{2 \text { peak }}\right)$, they did not compensate by accelerating muscle $\mathrm{O}_{2}$ extraction (Figure 1). This raised questions regarding the capacity of CF skeletal muscle and/or its microcirculation to extract and use $\mathrm{O}_{2}$.

We took this further to, for the first time, study $\mathrm{O}_{2}$ transport and utilisation during physical activity that more closely reflects activities of daily living, i.e. bouts of constant work rate exercise (Saynor et al., 2016). Our finding that the phase II and overall $\mathrm{VO}_{2}$ on-kinetic response, a proxy for muscle $\mathrm{O}_{2}$ consumption, was slowed during very heavy- but not moderate-intensity exercise supported the notion that impaired muscle oxidative metabolism in children and adolescents with mild-to-moderate CF lung disease is exercise intensitydependent. Furthermore, we observed a significant reduction in the arterio-venous $\mathrm{O}_{2}$ content difference $\left(\mathrm{C}_{(\mathrm{a}-\mathrm{v})} \mathrm{O}_{2}\right)$, a measure of the amount of $\mathrm{O}_{2}$ taken up by the tissues - with a greater difference reflecting greater $\mathrm{O}_{2}$ extraction-, during the time interval corresponding to the phase II $\mathrm{VO}_{2}$ response. $\Delta \mathrm{C}_{(\mathrm{a}-\mathrm{v})} \mathrm{O}_{2}$ was also associated with the $\dot{\mathrm{V}}_{2}$ kinetic response during very heavy-intensity exercise in CF but not healthy controls, suggesting a mechanistic link and further supporting the notion that pwCF are principally limited by intramuscular factors, which limit $\mathrm{O}_{2}$ extraction and/or utilisation during exercise. Physiological compensation for this does however only appear viable at lower exercise intensities in milder disease, a finding that likely reflects the greater physiological challenge more intense exercise poses to mitochondrial aerobic metabolism.

One of our laboratories was also the first to use NIRS during a non-invasive recovery kinetics test evaluating skeletal muscle oxidative capacity in pwCF. Measuring the recovery rate of oxygen consumption of the $m$. vastus lateralis following $15 \mathrm{~s}$ of electrical stimulation $(4 \mathrm{~Hz})$ and subsequent repeated transient arterial occlusions (Erickson et al., 2015), we identified impaired skeletal muscle oxidative capacity in pwCF. A significant inverse relationship between skeletal muscle oxidative capacity and age was also observed in pwCF, but not 
healthy individuals, indicating that an age-related reduction in skeletal muscle oxidative capacity may be evident in CF.

Manipulating $\mathrm{O}_{2}$ delivery and extraction can also provide insight into the locus of exercise limitation. Various strategies exist, such as reducing the work of breathing during exercise via inspiration of hyperoxic gas. This should increase systemic $\mathrm{O}_{2}$ delivery, however early evidence in pwCF suggested that whilst $\mathrm{O}_{2}$ supplementation could normalise $\mathrm{O}_{2}$ saturation, this does not improve $\dot{\mathrm{V}} \mathrm{O}_{2 \text { peak }}$ (Nixon et al., 1990) or $\dot{\mathrm{V}} \mathrm{O}_{2}$ kinetics (Kusenbach et al., 1999). Another established experimental manipulation to reveal the role of central limiting factors is 'priming' exercise. Priming exercise has been shown to speed $\mathrm{V}_{2}$ kinetics in individuals, through enhanced muscle $\mathrm{O}_{2}$ availability, microvascular oxygenation and activation of the mitochondrial pyruvate dehydrogenase complex, in addition to improved muscle perfusion secondary to the vasodilatory effects of metabolic acidaemia. If priming exercise increased systemic $\mathrm{O}_{2}$ delivery via an increase in cardiac output in pwCF and improved the matching of $\mathrm{O}_{2}$ delivery-to-utilisation (i.e. reduced $\Delta[\mathrm{HHB}] / \dot{\mathrm{VO}}_{2}$ ), this would provide insight into the $\mathrm{O}_{2}$ delivery-dependence of aerobic fitness. However, currently unpublished data from our laboratory has revealed that priming exercise cannot increase cardiac output in youth with $\mathrm{CF}$ and did not accelerate $\dot{\mathrm{V}} \mathrm{O}_{2}$ kinetics. These observations further support the mechanistic role of localised intramuscular consequences of $\mathrm{CF}$ to limit exercise tolerance rather than central factors.

\section{TREATMENT OPTIONS FOR EXERCISE IN TOLERANCE IN PEOPLE WITH CYSTIC FIBROSIS}

In the cases where an $\mathrm{O}_{2}$ utilisation limitation to $\mathrm{V}_{2}$ kinetics is responsible for the reduced tolerance to aerobic exercise in pwCF, for example those with preserved lung function, exercise training to improve mitochondrial function, such as high-intensity interval training (HIIT), might be particularly beneficial. Reuveny and colleagues (Reuveny et al., 2020) recently provided the first evidence that 8 weeks HIIT can accelerate $\dot{\mathrm{VO}}_{2}$ kinetics and improve exercise tolerance in $\mathrm{pwCF}$. Although central and peripheral function were not independently assessed; considering that HIIT is particularly suited to inducing peripheral adaptations, their findings provide further evidence that a peripherally-based factor is principally rate limiting in pwCF. 
Aside from exercise, Sildenafil, a phosphodiesterase type 5 inhibitor, has shown promising results. Sildenafil can improve CFTR chloride channel function (Lubamba et al., 2011), inflammation (Taylor-Cousar et al., 2015), and blood flow to and within muscles (Sperandio et al., 2012). Data from our team recently reported improvements in vascular function (Rodriguez-Miguelez et al., 2018) and exercise capacity after a single dose, which was maintained throughout 4 weeks of treatment (Rodriguez-Miguelez et al., 2019). A more indepth analysis supports that a sub-acute treatment with sildenafil was able to improve skeletal muscle $\mathrm{O}_{2}$ extraction and utilisation in pwCF during maximal exertion (Figure 1). Not only does this data provide further evidence regarding the vascular and intramuscular involvement in exercise limitation characterising pwCF, but importantly offers a therapeutic strategy to, at least in part, treat this.

A pharmacological 'helping hand' could also be administered. We have previously demonstrated in a small case series that Ivacaftor, a CFTR modulator therapy, improved aerobic fitness in pwCF with the G551D mutation (Saynor et al., 2014a), due to improvements in both $\mathrm{O}_{2}$ delivery and extraction in the absence of training. Currently unpublished data from one of our laboratories has more recently shown that ElexacaftorTezacaftor-Ivacaftor, can improve exercise capacity in adolescents with CF, with early indicators suggesting improvements in $\mathrm{O}_{2}$ transport/utilisation in the exercising muscle independent of changes in ventilatory function.

\section{SUMMARY AND CONSIDERATIONS}

As highlighted in this review, exercise intolerance in $\mathrm{pwCF}$ is multifactorial with a mechanistic basis that shifts with age and disease severity. To date ventilatory, cardiovascular and intramuscular abnormalities have been reported and we suggest that mechanisms of exercise intolerance is likely distributed differently and dependent on disease severity. At lower intensities of exercise and in mild CF lung disease, physiological compensation is possible. However, with more severe dysfunction and more intense exercise, a pharmacological 'helping hand' may be needed to improve exercise capacity. 


\section{REFERENCES}

Causer AJ, Shute JK, Cummings MH, Shepherd AI, Gruet M, Costello JT, Bailey S, Lindley M, Pearson C \& Connett G (2020). Circulating biomarkers of antioxidant status and oxidative stress in people with cystic fibrosis: A systematic review and meta-analysis. Redox biology 32, 101436.

Chen H, Liu LL, Ye LL, McGuckin C, Tamowski S, Scowen P, Tian H, Murray K, Hatton WJ \& Duan D (2004). Targeted inactivation of cystic fibrosis transmembrane conductance regulator chloride channel gene prevents ischemic preconditioning in isolated mouse heart. Circulation 110, $700-704$.

Divangahi M, Balghi H, Danialou G, Comtois AS, Demoule A, Ernest S, Haston C, Robert R, Hanrahan JW \& Radzioch D (2009). Lack of CFTR in skeletal muscle predisposes to muscle wasting and diaphragm muscle pump failure in cystic fibrosis mice. PLoS genetics 5, e1000586.

Eising JB, van der Ent CK, Teske AJ, Vanderschuren MM, Uiterwaal CS \& Meijboom FJ (2018). Young patients with cystic fibrosis demonstrate subtle alterations of the cardiovascular system. Journal of Cystic Fibrosis 17, 643-649.

Erickson ML, Seigler N, McKie KT, McCully KK \& Harris RA (2015). Skeletal muscle oxidative capacity in patients with cystic fibrosis. Experimental physiology 100, 545-552.

Giacchi V, Rotolo N, Amato B, Di Dio G, Betta P, La Rosa M, Leonardi S \& Sciacca P (2015). Heart involvement in children and adults with cystic fibrosis: correlation with pulmonary indexes and inflammation markers. Heart, Lung and Circulation 24, 1002-1010.

Gruet M, Troosters T \& Verges S (2017). Peripheral muscle abnormalities in cystic fibrosis: etiology, clinical implications and response to therapeutic interventions. Journal of Cystic Fibrosis 16, 538-552.

Hart P, Warth JD, Levesque PC, Collier ML, Geary Y, Horowitz B \& Hume JR (1996). Cystic fibrosis gene encodes a cAMP-dependent chloride channel in heart. Proceedings of the National Academy of Sciences 93, 6343-6348.

Khalaf MM, Scott-Ward, T.S., Causer, A.J., Saynor, Z.L., Shepherd, A.I., Gorecki, D., Lewis, A., Laight, D. and Shute, J.K. (2020). Cystic fibrosis transmembrane conductance regulator (CFTR) in human lung microvascular endothelial cells controls oxidative stress, reactive oxygen-mediated cell signalling and inflammation. Frontiers in Physiology 11, 879.

Kusenbach G, Wieching R, Barker M, Hoffmann U \& Essfeld D (1999). Effects of hyperoxia on oxygen uptake kinetics in cystic fibrosis patients as determined by pseudo-random binary sequence exercise. European journal of applied physiology and occupational physiology 79, 192-196. 
Labombarda F, Saloux, E., Brouard, J., Bergot, E. and Milliez, P. (2016). Heart involvement in cystic fibrosis: A specific cystic fibrosis-related myocardial changes? Respiratory Medicine and Research 118, 31-38.

Lamhonwah AM, Bear CE, Huan LJ, Chiaw PK, Ackerley CA \& Tein I (2010). Cystic fibrosis transmembrane conductance regulator in human muscle: dysfunction causes abnormal metabolic recovery in exercise. Annals of neurology 67, 802-808.

Linsdell P \& Hanrahan JW (1998). Glutathione permeability of CFTR. American Journal of Physiology-Cell Physiology 275, C323-C326.

Lubamba B, Lebacq J, Reychler G, Marbaix E, Wallemacq P, Lebecque P \& Leal T (2011). Inhaled phosphodiesterase type 5 inhibitors restore chloride transport in cystic fibrosis mice. European Respiratory Journal 37, 72-78.

Nixon PA, Orenstein DM, Curtis SE \& Ross EA (1990). Oxygen Supplementation during Exercise in Cystic Fibrosis1-3. Am Rev Respir Dis 142, 807-811.

Poore S, Berry B, Eidson D, McKie KT \& Harris RA (2013). Evidence of vascular endothelial dysfunction in young patients with cystic fibrosis. Chest 143, 939-945.

Reuveny R, DiMenna FJ, Gunaratnam C, Arad AD, McElvaney GN, Susta D, Peled M \& Moyna NM (2020). High-intensity interval training accelerates oxygen uptake kinetics and improves exercise tolerance for individuals with cystic fibrosis. BMC Sports Science, Medicine and Rehabilitation 12, 1-13.

Rodriguez-Miguelez P, Ishii H, Seigler N, Crandall R, Thomas J, Forseen C, McKie KT \& Harris RA (2019). Sildenafil improves exercise capacity in patients with cystic fibrosis: a proof-ofconcept clinical trial. Therapeutic Advances in Chronic Disease 10, 2040622319887879.

Rodriguez-Miguelez P, Lee N, Tucker MA, Csányi G, McKie KT, Forseen C \& Harris RA (2018). Sildenafil improves vascular endothelial function in patients with cystic fibrosis. American Journal of Physiology-Heart and Circulatory Physiology 315, H1486-H1494.

Rodriguez-Miguelez P, Seigler N, Ishii H, Crandall R, McKie KT, C F \& Harris RA (In Press). Exercise Intolerance in Cystic Fibrosis: Importance of Skeletal Muscle. Medicine \& Science in Sports \& Exercise.

Rodriguez-Miguelez P, Thomas J, Seigler N, Crandall R, McKie KT, Forseen C \& Harris RA (2016). Evidence of microvascular dysfunction in patients with cystic fibrosis. American Journal of Physiology-Heart and Circulatory Physiology 310, H1479-H1485.

Saynor ZL, Barker AR, Oades PJ \& Williams CA (2014a). The effect of ivacaftor in adolescents with cystic fibrosis (G551D mutation): an exercise physiology perspective. Pediatric Physical Therapy 26, 454-461. 
Saynor ZL, Barker AR, Oades PJ \& Williams CA (2014b). Impaired aerobic function in patients with cystic fibrosis during ramp exercise. Medicine and Science in Sports \& Exercise 46, 22712278.

Saynor ZL, Barker AR, Oades PJ \& Williams CA (2016). Impaired pulmonary V O2 kinetics in cystic fibrosis depend on exercise intensity.

Sellers ZM, Kovacs A, Weinheimer CJ \& Best PM (2013). Left ventricular and aortic dysfunction in cystic fibrosis mice. Journal of Cystic Fibrosis 12, 517-524.

Sperandio PA, Oliveira MF, Rodrigues MK, Berton DC, Treptow E, Nery LE, Almeida DR \& Neder JA (2012). Sildenafil improves microvascular O2 delivery-to-utilization matching and accelerates exercise $\mathrm{O} 2$ uptake kinetics in chronic heart failure. American Journal of Physiology-Heart and Circulatory Physiology 303, H1474-H1480.

Taylor-Cousar J, Wiley C, Felton L, Clair CS, Jones M, Curran-Everett D, Poch K, Nichols D, Solomon G \& Saavedra M (2015). Pharmacokinetics and tolerability of oral sildenafil in adults with cystic fibrosis lung disease. Journal of Cystic Fibrosis 14, 228-236.

Tousson A, Van Tine BA, Naren AP, Shaw GM \& Schwiebert LM (1998). Characterization of CFTR expression and chloride channel activity in human endothelia. American Journal of Physiology-Cell Physiology 275, C1555-C1564. 


\section{ACKNOWLEDGEMENTS}

We apologise to all authors who contributed to this field but could not be cited/recognised in this article due to space limitations.

\section{COMPETING INTERESTS}

None to declare

\section{AUTHOR CONTRIBUTIONS}

All authors contributed conceptually to the concepts detailed in this manuscript and the drafting of the article. All authors read and approved the final version of the article and agree to be accountable for all aspects of the work in ensuring that questions related to the accuracy or integrity of any part of the work are appropriately investigated and resolved. All persons designated as authors qualify for authorship, and all those who qualify for authorship are listed.

\section{ORCID}

Zoe L. Saynor: https://orcid.org/0000-0003-0674-8477

Mathieu Gruet:

Paula Rodriguez-Miguelez: https://orcid.org/0000-0003-3582-4483

Ryan A. Harris: 


\section{FIGURES}
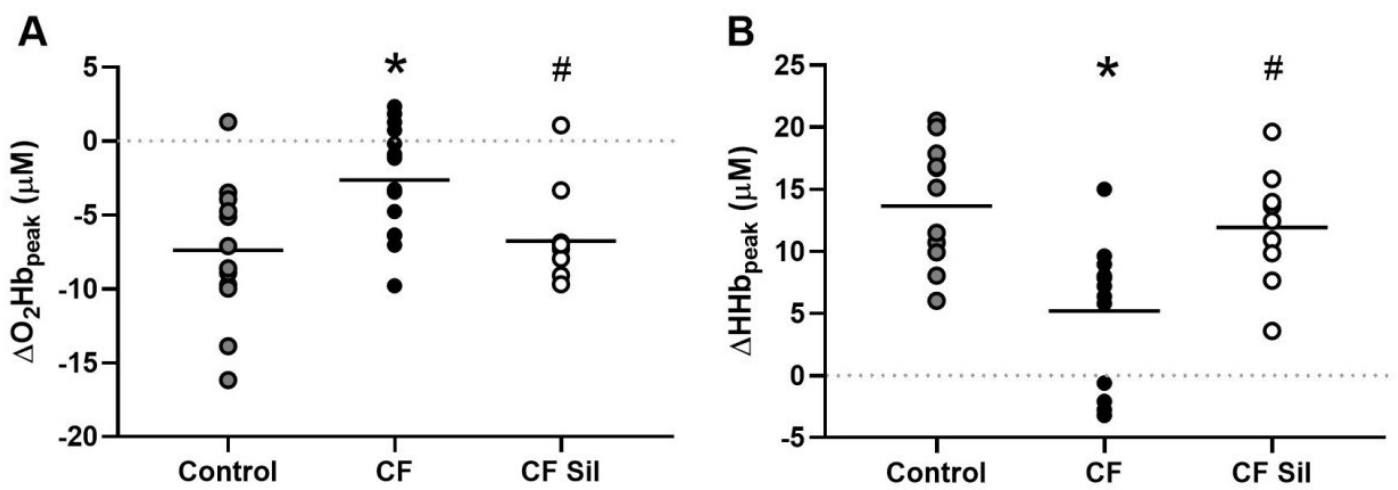

Figure 1. Skeletal muscle $\mathrm{O}_{2}$ utilisation during peak exercise. Change in oxygenated haemoglobin $\left(\Delta \mathrm{O}_{2} \mathrm{Hb}\right.$; panel $\left.\mathrm{A}\right)$ and deoxygenated haemoglobin $(\Delta \mathrm{HHb}$; panel $\mathrm{B})$ in healthy controls (grey circles), people with cystic fibrosis (CF) before (black circles) and following four weeks of sildenafil (SIL, white circles) from rest to peak $\mathrm{O}_{2}$ consumption. All measures are expressed as individual data and overall group means. All statistical analyses were performed using SPSS Version 25 (SPSS Inc., Chicago, IL, USA), and significance was set at $p<0.05$. Independent group $t$ - tests were used to compare controls and people with $\mathrm{CF}$ (pwCF). Paired $t$ - tests were performed to identify differences between baseline and treatment with sildenafil within the pwCF. *Significant difference between pwCF and controls. \#Significant difference $(p<0.05)$ between baseline and four weeks of treatment with sildenafil in pwCF. Figure represents more detailed analysis of data derived from Rodriguez Miguelez et al. (Rodriguez-Miguelez et al., In Press). 